\title{
Prominent and fruitful development of orthopaedic research in China
}

\author{
Wei Chen ${ }^{1,2,3} \cdot$ Yingze Zhang ${ }^{1,2,3,4}$
}

Published online: 24 February 2018

(C) SICOT aisbl 2018

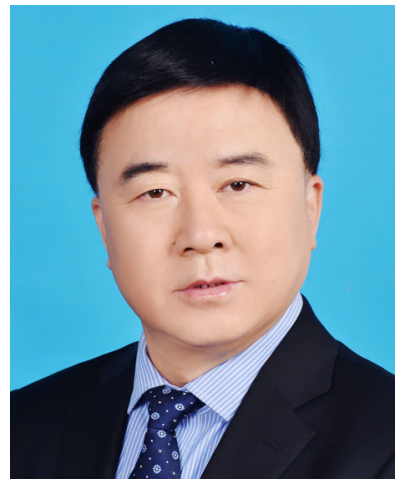

Yingze Zhang

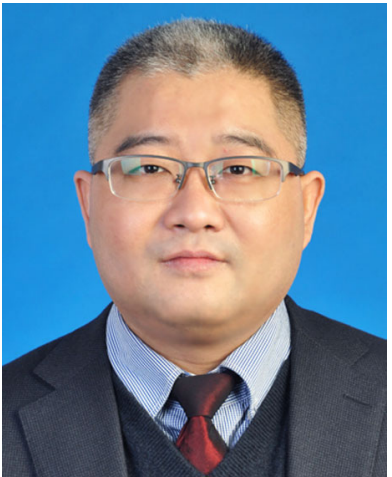

Wei Chen
With the remarkable and fruitful development of the Chinese society and economy, the government has injected significant capital into both clinical practice and medical scientific research striving for continuous improvement of the health care system. Great achievements have been made in all subspecialties of medicine in the last decades. China's orthopaedics research has also made rapid growth in terms of both quality and quantity of scientific publications [1]. International orthopaedics dedicates a special issue focused on the introduction of recent achievements of orthopaedic research in China. We

Yingze Zhang

dryzzhang@126.com

Wei Chen

surgeonchenwei@126.com

1 Department of Orthopedic Surgery, The Third Hospital of Hebei Medical University, Shijiazhuang, Hebei 050051, People's Republic of China

2 Key laboratory of biomechanics of Hebei Province, Shijiazhuang, Hebei 050051, People's Republic of China

3 Hebei Institute of Orthopaedics and Traumatology, The Third Hospital of Hebei Medical University, Shijiazhuang, Hebei 050051, People's Republic of China

4 Chinese Academy of Engineering, Beijing 100088, People's Republic of China are very honoured and privileged to take part in the organization of such a special issue covering a large range of orthopaedic subspecialties, including epidemiology, traumatic fractures, osteoarthritis, spinal disorders, infections, and orthopaedic oncology.

Traumatic fractures are always a hotspot issue of orthopaedic care and research. Our team has conducted for many years an epidemiologic investigation of fractures nationwide in China, developed in two parts [2-7]. In the first part, we collected 497,089 fractures from 83 hospitals across China and reported the clinical characteristics and statistical overview of fracture location, segment, and AO/OTA fracture type. In part II, we reported the national incidence, distribution, and risk factors of traumatic fractures based upon a nationally representative population-based study of 512,187 individuals. In this special issue, the epidemiological characters of tibial plateau fractures and clavicle fractures are reported $[2,3]$. In addition, Wang $\mathrm{H}$ et al. investigated the incidence and pattern of traumatic fractures resulting from motor vehicle collisions among children and adolescents ( $\leq 18$ years old). They found that patients in the age group between 15 and 18 years as well as the drivers had the highest frequencies of both nerve injury and early complications and that craniofacial and spinal fractures were independent risk factors for nerve injury [8]. Epidemiological studies of traumatic fractures can be used to improve the comprehensive understanding of traumatic 
fractures of each bone and various patients and formulate injury control policies and interventions.

Elbow fractures are a common injury, accounting for up to $30 \%$ of all upper extremity fractures [9]. Among them, lateral condyle fractures, which are easily misdiagnosed as total distal humeral physeal fracture or a primary dislocation of the elbow, make up $17 \%$ of distal humeral fractures in children [10]. Through a comparative study of the lateral condyle fracture with or without posteromedial elbow dislocation, Lan et al. reported that Milch type II fractures of lateral humeral condyle with concomitant posteromedial elbow dislocation extended to the lower medial part of the trochlea, and initial recognition of this rare injury is demanding to regain satisfactory functional recovery [10]. An important feature to evaluate the functional outcomes was the joint stiffness that is a well-known complication of elbow fractures. Zheng et al. performed a retrospective study involving patients with post-traumatic elbow stiffness and found that high-energy injuries were associated with the development of severe elbow stiffness [9]. Cui et al. reported that the ranges of motion of the elbow can be improved using open release combined with external fixation and that stability can be strengthened using ligament repair in patients with the terrible triad injury, no matter if the radial head was resected or replaced in previous treatments [11].

Minimally invasive reduction and fixation of pelvic and acetabular fractures are of technique demand but with a good prospect in clinical practice. Three-dimensional navigationguided percutaneous screw fixation was applied in the treatment of both nondisplaced and displaced pelvi-acetabular fractures. Fifty-nine sacroiliac, 45 retrograde anterior column, 34 supra-acetabular, three antegrade posterior-column, and two subcristal percutaneous screw fixation were inserted exactly in 38 consecutive patients without immediate or early surgical complications, and uneventful fracture union as well as satisfied functional recovery was reported at follow-ups [12]. Wang et al. treated 29 patients with type $\mathrm{B} 2$ and $\mathrm{C} 1$ posterior pelvic ring fractures in a minimally invasive fashion with the use of pedicle screws connected to a transverse rod [13]. In this study, the posterior-ring fracture reduction was evaluated as excellent and good rate in $89.7 \%$ cases according to Tornetta and Matta standard, and the function outcome was rated as excellent and good in 26 cases based upon Majeed scores. This technique may be a good alternative in patients who do not qualify for sacroiliac screw fixation. A biomechanical study comparing different fixation techniques for acetabular posterior wall fractures was also reported in this issue, which reveals that reconstruction plate and compression screw fixation with a plate placed lateral to the screws may address acetabular posterior wall fractures in a more stable and appropriate manner than techniques using only plates or screws [14].

Hip fracture is a major cause of morbidity and mortality in the elderly, which has been advocated to be treated at the earliest possible setting with the use of internal fixation, total hip arthroplasty (THA), or other indicated procedures. However, small delays in surgery do not increase the mortality rate of elderly patients [15]. How to reduce pain and improve the functional recovery after THA? A randomized controlled trial conducted by Lei and his colleagues demonstrated that the administration of two low-dose $(10 \mathrm{mg})$ peri-operative dexamethasone can effectively reduce the level of C-reactive protein and interleukin-6, pain during mobilization, and the incidence of post-operative nausea and vomiting, ameliorate post-operative fatigue, and enhance the range of motion of hip joint without increasing the risk of infection and gastrointestinal hemorrhage [16]. THA is also an effective treatment for patients with ankylosing spondylitis and hip joint fusion. The improvements in joint function and self-care ability after THA are the decisive factors in determining patient satisfaction for such patients [17]. Periprosthetic joint infection (PJI) is a serious complication after total joint arthroplasty and continues to be a great challenge for both surgeons and patients, especially for immunocompromised patients. For immunocompromised patients with PJI, high rate of recurrent infection was observed in a case series of 29 patients treated by one-stage revision or debridement, antibiotics and implant retention, and Cao L et al. suggested that more attention should be paid to the concept of disease control rather than cure for such a population [18].

Researchers have made encouraging progress in the basic research and clinical treatment of knee osteoarthritis (KOA). It is found that leptin was higher and adiponection was lower in MetS-OA patients [19], potentially providing a biological direction on KOA diagnosis. Jang et al. [20] reported that preand post-operative hip-knee-angle, post-operative American Knee Society (AKS) and the Western Ontario and McMaster Universities (WOMAC) score were significant predictors for subjective satisfaction in patients undergoing opening-wedge high tibial osteotomy. Mini-subvastus approach could offer faster recovery, less pain, and shorter hospital stays without compromising the principles of proper prosthesis position and limb alignment compared with the medial parapatellar approach of TKA [21]. Zha et al. [22] retrospectively analyzed the relationship between chondromalacia patellae (CMP) and post-operative anterior knee pain (AKP). They found that CMP has no influence on the incidence of AKP following TKA without patellar resurfacing. Valgus cut angle (VCA) and the femoral entry point should be determined individually for each case. Yue et al. [23] introduced an effective method that can accurately determine the distal femoral VCA in the valgus knee arthroplasty. It is demonstrated that keeping the knee in flexion position combined with topical and intravenous tranexamic acid application could significantly reduce postoperative blood loss and the transfusion rate in patients undergoing primary unilateral TKA [24].

Lumbar disc herniation (LDH) and congenital scoliosis (CS) are common encountered spine disorders. LDH is 
thought to be a disease mainly occurring in young patients. Tao et al. found that laminae defects of L5 may be a congenitally potential risk factor leading to lower LDH in the young [25]. Through a comprehensive comparison of therapeutic efficacy of radiofrequency target disk decompression and nucleoplasty for $\mathrm{LDH}$, Nie et al. found that nucleoplasty allowed patients to have a short hospitalization and operation time which may accelerate the rate of rehabilitation [26]. CS frustrates both young and elder patients. It was reported that hypoxia aggravated the proportion and severity of rib deformities and affected the development of spinal cord, especially in patients who tend to have higher proportion and more severe of rib deformities in high-altitude geographic regions [27]. The most commonly used internal fixation for correction of spinal disorder is pedicle screw. Several techniques for pedicle screw placement, such as Roy-Camille technique, were used in clinic, but the rate of misplacement of pedicle screws in lumbar spine is still high. A new insertion site located at the outer edge of the superior facet joint and $4 \mathrm{~mm}$ downward from the upper border of processus transversus were introduced and exhibited safety and effectiveness in clinical application [28].

Innovative ideas and their realization and application in clinics are the inexhaustible driving force for development of orthopaedic surgery. For refractory bone sarcomas that might lead to catastrophic consequence, innovative ideas are especially important and potentially produce a smooth unexpected favourable result. In Lou' pilot study of five patients with primary distal tibial sarcomas (two Ewing's sarcomas and three osteosarcomas) [29], resection arthrodesis using distraction osteogenesis then plating as a hybrid surgical technique was applied to obtain the favourable functional results of limb salvage, with the mean functional score of $88 \%$ (83$90 \%$ ) according to the rating system of the Musculoskeletal Tumour Society. Similarly, emergence of computer and 3D printing technique makes the personal design of prosthesis a reality, even for the malignant bone tumour at peri-acetabulum. Wang et al. treated 11 patients with peri-acetabular malignant bone tumours with personalized 3D-printed hemipelvic prostheses after en bloc resection, and after an average of 15.5-month follow-up, no local tumour recurrence was observed and most of the patients could obtain a relatively satisfied result (19.2 based on MSTS-93 score) [30]. It is believed that successful application of the innovative idea and advanced technique could provide a promising implication and alternative treatment for patients with malignant bone tumours, in the future clinical practices.

The treatment of large post-traumatic bone defects and shortening with or without deformity remains a significant challenge in the field of orthopaedics. Zhang et al. applied double-level bone transport technique, a modified procedure of traditional Ilizarov single-level bone transport technique, to treat tibial fractures with large bone defects [31]. Excellent and good functional outcomes were achieved in all cases with reduced bone transport time, time in frame, and total treatment time. Ruan et al. treated humeral shortening and proximal varus angular deformity using a combined protocol of acute deformity correction and gradual lengthening with the monorail external fixator, which also witnessed obvious functional improvement after surgery and post-operative therapy [32].

Meta-analysis is high level of evidence in addressing the inconsistent findings and unfeasibility presented in the original scientific researches. It is reported that majority of the Chinese articles with most citations were systematic reviews and/or meta-analysis (47\%) for the last five years [1]. In this study, there are three meta-analysis papers. Lu et al. presented a systematic review of ten recent studies regarding the operative techniques for osteochondritis dissecans (OCD) of the humeral capitellum [33]. They found that arthroscopic lesion debridement with fragment fixation can treat unstable OCD as good as, if not better than open procedure. Sun Q et al. performed a systemic review and meta-analysis to compare the clinical outcome and complications of displaced proximal humeral fractures treated with locking plates versus intramedullary nails [34]. Similar functional outcomes and total complication rate were comparable between both procedures without evidence showing one treatment superior to the other. Wang et al. [35] reported in their meta-analysis that asymptomatic bacteriuria increases the risk of prosthetic joint infection; however, current evidence does not support systematic antibiotherapy prior to joint arthroplasty and screening for asymptomatic bacteriuria.

In addition to the above-introduced articles, there are several other exciting studies included in this issue. Zhao et al. reported their initial experience of the newly designed antegrade interlocking angle-stable intramedullary nail combined with half-threaded cancellous screws in the treatment of type C (AO/OTA classification) distal femoral fractures [36]. $\mathrm{Li}$ et al. verified that continuous vibration therapy and seven day intermittent vibration therapy pattern can achieve better improvement of fracture union [37]. Yang et al. presented their imaging study using computed tomography scans and threedimensional reconstruction images, which quantified glenoid morphology and define the safe zone for protecting the suprascapular nerve during baseplate fixation in reverse shoulder arthroplasty [38]. Li et al. conducted a retrospective study and identified acetabular index as the best predictor of late residual acetabular dysplasia after closed reduction in developmental dysplasia of the hip [39].

This special issue also includes six papers classified as Letters to the Editor [40-45]. We appreciate the readers' interests and disputes on the studies published in International Orthopaedics. Truth can be gleaned from lively debates, and consensus is usually formed in the midst of discussions and arguments. 


\section{References}

1. Sun J, Guo Y, Scarlat M, Lv G, Yang XG, Hu YC (2017) Bibliometric study of the Orthopaedic publications from China. Int Orthop. https://doi.org/10.1007/s00264-018-3828-8

2. Chen W, Zhu Y, Liu S, Hou Z, Zhang X, Lv H, Zhang Y (2017) Demographic and socioeconomic factors influencing the incidence of clavicle fractures, a national population-based survey of 512187 individuals. Int Orthop. https://doi.org/10.1007/s00264-018-3815-0

3. Yuwen P, Lv H, Chen W, Wang Y, Yu Y, Hao J, Liu S, Zhang T, Feng C, Guo J, Yin B, Zhang Y (2017) Age-, gender- and AO type specific clinical characters of adult tibial plateau fractures in 83 hospitals in China. Int Orthop. https://doi.org/10.1007/s00264018-3769-2

4. Chen W, Lv H, Liu S, Liu B, Zhu Y, Chen X, Yang G, Liu L, Zhang T, Wang H, Yin B, Guo J, Zhang X, Li Y, Smith D, Hu P, Sun J, Zhang Y (2017) National incidence of traumatic fractures in China: a retrospective survey of 512187 individuals. Lancet Glob Health 5(8):e807-e817. https://doi.org/10.1016/S2214-109X(17)30222-X

5. Zhang Y (ed) (2014) Clinical epidemiology of Orthopaedic trauma, 1 st edn. Thieme, Stuttgart

6. Zhang Y (ed) (2016) Clinical epidemiology of Orthopaedic trauma, 2nd edn. Thieme, Stuttgart

7. Tian Y, Zhu Y, Yin B, Zhang F, Liu B, Chen W, Zhang Y (2016) Age- and gender-specific clinical characteristics of acute adult spine fractures in China. Int Orthop 40(2):347-353. https://doi.org/10. 1007/s00264-015-3025-y

8. Wang H, Zhou Y, Liu J, Ou L, Zhao Y, Han J, Xiang L (2017) Traumatic fractures as a result of motor vehicle collisions in children and adolescents. Int Orthop. https://doi.org/10.1007/s00264018-3777-2

9. Zheng W, Liu J, Song J, Fan C (2017) Risk factors for development of severe post-traumatic elbow stiffness. Int Orthop. https://doi.org/ 10.1007/s00264-017-3657-1

10. Lan X, Dai M, Zhang B, Huang G (2017) Comparative study of lateral condyle fracture with or without posteromedial elbow dislocation in children. Int Orthop. https://doi.org/10.1007/s00264-0183795-0

11. Cui HM, Yu YL, He Y, Cheng Y, Liu JZ, Zheng W, Chen S, Fan CY (2017) Management of elbow stiffness after postoperative treatment of terrible triad elbow injury: maintaining mobility and stability using a combined protocol. Int Orthop. https://doi.org/10.1007/ s00264-017-3721-x

12. Chui KH, Chan CCD, Ip KC, Lee KB, Li W (2017) Threedimensional navigation-guided percutaneous screw fixation for nondisplaced and displaced pelvi-acetabular fractures in a major trauma centre. Int Orthop. https://doi.org/10.1007/s00264-017-3659-Z

13. Wang $\mathrm{H}, \mathrm{Fu} \mathrm{YH}, \mathrm{Ke} \mathrm{C}$, Zhuang Y, Zhang K, Wei X, Li Z, Lei JL, Zhang BF, Liu P (2017) Minimally invasive stabilisation of posterior pelvic ring instabilities with pedicle screws connected to a transverse rod. Int Orthop. https://doi.org/10.1007/s00264-017-3714-9

14. Wu X (2017) A biomechanical comparison of different fixation techniques for fractures of the acetabular posterior wall. Int Orthop. https://doi.org/10.1007/s00264-017-3728-3

15. Wong SHJ, Fang XC, Yee KHD, Wong TM, Pun CTT, Lau TW, Leung KLF (2017) Hip fracture time-to-surgery and mortality revisited: mitigating comorbidity confounding by effect of holidays on surgical timing. Int Orthop. https://doi.org/10.1007/s00264-017-3737-2

16. Lei YT, Xu B, Xie XW, Xie JW, Huang Q, Pei FX (2017) The efficacy and safety of two low-dose peri-operative dexamethasone on pain and recovery following total hip arthroplasty: a randomized controlled trial. Int Orthop. https://doi.org/10.1007/s00264-0173537-8

17. Ding L, Gao YH, Li YR, Liu JG, Li SQ, Qi X (2017) Determinants of satisfaction following total hip arthroplasty in patients with ankylosing spondylitis. Int Orthop. https://doi.org/10.1007/ s00264-017-3589-9

18. Ji B, Zhang X, Xu B, Ren J, Guo W, Mu W, Cao L (2017) The fate of immunocompromised patients in the treatment of chronic periprosthetic joint infection: a single-centre experience. Int Orthop. https://doi.org/10.1007/s00264-018-3763-8

19. Dong N, Gao YH, Liu B, Zhao CW, Yang C, Li SQ, Liu JG, Qi X (2017) Differential expression of adipokines in knee osteoarthritis patients with and without metabolic syndrome. Int Orthop. https:// doi.org/10.1007/s00264-018-3761-x

20. Han SB, Lee JH, Kim SG, Cui CG, Suh DW, Lee SY, Jang KM (2017) Patient-reported outcomes correlate with functional scores after opening-wedge high tibial osteotomy: a clinical study. Int Orthop. https://doi.org/10.1007/s00264-017-3614-z

21. Li Z, Cheng W, Sun L, Yao Y, Cao Q, Ye S, Qi L, Xu S, Wu X, Jing J (2017) Mini-subvastus versus medial parapatellar approach for total knee arthroplasty: a prospective randomized controlled study. Int Orthop. https://doi.org/10.1007/s00264-017-3703-z

22. Zha GC, Feng S, Chen XY, Guo KJ (2017) Does the grading of chondromalacia patellae influence anterior knee pain following total knee arthroplasty without patellar resurfacing? Int Orthop. https://doi.org/10.1007/s00264-017-3658-0

23. Tan H, Wang Y, Long T, Nie B, Mao Z, Yue B (2017) How to accurately determine the distal femoral valgus cut angle in the valgus knee arthroplasty. Int Orthop. https://doi.org/10.1007/s00264018-3778-1

24. Zeng Y, Si H, Li C, Wu Y, Shen B (2017) Effect of knee flexion position and combined application of tranexamic acid on blood loss following primary total knee arthroplasty: a prospective randomized controlled trial. Int Orthop. https://doi.org/10.1007/s00264018-3808-z

25. Tao S, Jin L, Hou Z, Zhang W, Chen T, Zhang Y (2017) A new radiographic feature of lower lumbar disc herniation in young patients. Int Orthop. https://doi.org/10.1007/s00264-017-3723-8

26. Nie HY, Qi YB, Li N, Wang SL, Cao YX (2017) Comprehensive comparison of therapeutic efficacy of radiofrequency target disc decompression and nucleoplasty for lumbar disc herniation: a five year follow-up. Int Orthop. https://doi.org/10.1007/s00264-0173661-5

27. Hou D, Kang N, Yin P, Hai Y (2017) Abnormalities associated with congenital scoliosis in high-altitude geographic regions. Int Orthop. https://doi.org/10.1007/s00264-018-3805-2

28. Zhang D, Gao X, Jiang J, Shen Y, Ding W, Cui H (2017) Safe placement of pedicle screw in lumbar spine with minimum three year follow-up: a case series and technical note. Int Orthop. https:// doi.org/10.1007/s00264-018-3806-1

29. Lou TF, Li H, Chai YM, Wang CY, Liu SH, Hamushan M, Wu F, Cai WJ, Han P (2017) Resection arthrodesis using distraction osteogenesis then plating as a hybrid surgical technique for the management of bone sarcomas of the distal tibia. Int Orthop. https://doi. org/10.1007/s00264-018-3811-4

30. Wang B, Hao Y, Pu F, Jiang W, Shao Z (2017) Computer-aided designed, three dimensional-printed hemipelvic prosthesis for periacetabular malignant bone tumour. Int Orthop. https://doi.org/10. 1007/s00264-017-3645-5

31. Zhang Y, Wang Y, Di J, Peng A (2017) Double-level bone transport for large post-traumatic tibial bone defects: a single centre experience of sixteen cases. Int Orthop. https://doi.org/10.1007/s00264017-3684-y

32. Ruan H, Zhu Y, Liu S, Kang Q (2017) Humeral lengthening and proximal deformity correction with monorail external fixator in young adults. Int Orthop. https://doi.org/10.1007/s00264-0173688-7

33. Lu Y, Li YJ, Guo SY, Zhang HL (2017) Is there any difference between open and arthroscopic treatment for osteochondritis dissecans (OCD) of the humeral capitellum: a systematic review 
and meta-analysis. Int Orthop. https://doi.org/10.1007/s00264-0183768-3

34. Sun Q, Ge W, Li G, Wu J, Lu G, Cai M, Li S (2017) Locking plates versus intramedullary nails in the management of displaced proximal humeral fractures: a systematic review and meta-analysis. Int Orthop. https://doi.org/10.1007/s00264-017-3683-Z

35. Wang C, Yin D, Shi W, Huang W, Zuo D, Lu Q (2017) Current evidence does not support systematic antibiotherapy prior to joint arthroplasty in patients with asymptomatic bacteriuria-a meta analysis. Int Orthop. https://doi.org/10.1007/s00264-018-3765-6

36. Zhao Z, Li Y, Ullah K, Sapkota B, Bi H, Wang Y (2017) The antegrade angle-stable locking intramedullary nail for type-C distal femoral fractures: a thirty four case experience. Int Orthop. https:// doi.org/10.1007/s00264-017-3747-0

37. Li Y, Liu G, Yu J, Li C, Tan L, Hao B, Liu C, Lin J, Zhu D, Zhang X (2017) Effects of continuous or intermittent low-magnitude highfrequency vibration on fracture healing in sheep. Int Orthop. https:// doi.org/10.1007/s00264-018-3759-4

38. Yang Y, Zuo J, Liu T, Shao P, Wu H, Gao Z, Xiao J (2017) Glenoid morphology and the safe zone for protecting the suprascapular nerve during baseplate fixation in reverse shoulder arthroplasty. Int Orthop. https://doi.org/10.1007/s00264-017-3646-4

39. Li Y, Guo Y, Li M, Zhou Q, Liu Y, Chen W, Li J, Canavese F, Xu H (2017) Acetabular index is the best predictor of late residual acetabular dysplasia after closed reduction in developmental dysplasia of the hip. Int Orthop. https://doi.org/10.1007/s00264-0173726-5

40. Li H, Yang L (2017) Comment on article by parry et al.: Fungation in soft tissue sarcomas is associated with poor survival. Int Orthop. https://doi.org/10.1007/s00264-017-3694-9

41. Yan Z, Fang R, Liu T (2017) Is bone morphogenic protein-2 useful in revision total hip arthroplasty with acetabular defects? Int Orthop. https://doi.org/10.1007/s00264-017-3697-6

42. Chang SM, Hu SJ, Du SC, Ma Z, Xiong WF, Yao XZ (2017) Fourquadrant/column classification of tibial plateau fractures. Int Orthop. https://doi.org/10.1007/s00264-017-3733-6

43. Yan Z, Fang R, Liu T (2017) Partial component-retained two-stage reconstruction for chronic infection after uncemented total hip arthroplasty: good or bad? Int Orthop. https://doi.org/10.1007/ s00264-017-3748-z

44. Li HZ, Xu XH, Lu HD (2017) Letter regarding article by sun et al.: locking plates versus intramedullary nails in the management of displaced proximal humeral fractures: a systematic review and meta-analysis. Int Orthop. https://doi.org/10.1007/s00264-018-3786-1

45. Li Y, Wang J, Wang W (2017) Peri-operative antibiotic treatment of bacteriuria reduces early deep surgical site infections in geriatric patients with proximal femur fracture: is it related? Int Orthop. https://doi.org/10.1007/s00264-018-3784-3 